\title{
Identificação das veias e vasos portais dos lobos hepáticos de cães por meio da ultra-sonografia*
}

\section{Ultrasonographic identification of canine liver portal vessels and hepatic veins}

\section{Identificación de venas y vasos portales de los lóbulos hepáticos por medio de ultrasonografía}

\section{Annelise Baldacin Salgado; ${ }^{1}$ Arani Nanci Bomfim Mariana ${ }^{2}$}

Departamento de Cirurgia da Faculdade de Medicina Veterinária e Zootecnia da Universidade de São Paulo (FMVZ/USP). São Paulo, SP, Brasil

\section{Resumo}

Objetivo: Identificar as veias e vasos portais hepáticos de cães por meio da ultra-sonografia, avaliando a possibilidade de determinar seus lobos hepáticos com base naqueles vasos, de maneira semelhante à segmentação de fígados humanos. O exame ultra-sonográfico é um bom método para a avaliação do fígado, fornecendo dados sobre seu tamanho, forma e alterações do parênquima. Em cães, embora a visibilização e diferenciação entre as veias hepáticas e os vasos portais seja possível, são de difícil identificação anatômica e não há descrição na literatura de nenhum método que permita determinar os lobos hepáticos. Material e Método: Foram selecionados 15 cães vivos para a realização de exames ultra-sonográficos, sendo 10 hígidos e 5 com congestão venosa direita. Um cadáver foi utilizado para obtenção de molde vascular em vinil. Resultados e Conclusões: Os resultados foram registrados em vídeos e fotos, e mostraram que muitas das veias hepáticas e vasos do sistema porta hepático são de difícil identificação, o que permite concluir que o modelo humano de segmentação é inadequado para utilização em cães. Ainda assim, quando tal fenômeno é identificado, pode auxiliar na determinação dos lobos, pois representa importantes estruturas anatômicas de referência.

Palavras-chave: Anatomia. Fígado. Veias hepáticas. Ultrasonografia. Cães.

\footnotetext{
${ }^{1}$ Mestre em Anatomia dos Animais Domésticos e Silvestres pela FMVZ/USP. CRMV-SP 11664

${ }^{2}$ Professora Doutora do Departamento de Cirurgia da FMVZ/USP. CRMV-SP 1445

*Baseado em Dissertação de Mestrado apresentada à Faculdade de Medicina Veterinária e Zootecnia da Universidade de

São Paulo, 2002
} 


\section{Introdução}

A ultra-sonografia vem aos poucos ganhando maior importância em nosso meio, por tratar-se de um método não invasivo e seguro na deteç̧ão de inúmeras alteraç̃os, sendo de grande importância para a avaliação de órgãos como o fígado e, complementando o exame radiográfico.

Por ultra-sonografia, pode-se examinar a arquitetura interna do parênquima hepático, o sistema biliar, as estruturas perihepáticas e a sua vascularização (PARTINGTON, BILLER ${ }^{1}$ 1995; NYLAND et al., ${ }^{2}$ 1995; NYLAND, PARK, ${ }^{3}$ 1983; NYLAND, HAGER, $1985 ;{ }^{4}$ WRIGLEY, $\left.{ }^{5} 1985\right)$.

Pesquisadores como Nyland, Park ${ }^{3}$ (1983); Nyland, Hager ${ }^{4}$ (1985), Wrigley ${ }^{5}$ (1985) e Lamb ${ }^{6,7}$ $(1990,1991)$ realizaram estudos no sentido de diagnosticar doenças hepáticas por meio da ultrasonografia. Nyland e Park ${ }^{3}$ (1983) concluíram que a ultra-sonografia permite diagnosticar de maneira nãoinvasiva uma série de doenças que afetam o fígado dos animais, como alterações do parênquima e vasos sanguíneos, presença de massas neoplásicas primárias ou secundárias, cistos, abscessos, granulomas e alterações de vesícula e trato biliar. Concluíram também que a biópsia percutânea guiada por ultrasom dá maior segurança e sucesso na obtenção do material para o diagnóstico citológico. Mencionam ainda que, após o diagnóstico, a ultra-sonografia passa a ser um importante meio não invasivo de avaliação da terapia instituída.

Entretanto, ainda não é possível a demarcação dos lobos hepáticos do fígado de cães por faltarem descrições ultra-sonográficas, tanto da anatomia vascular como de outras estruturas, que poderiam servir como pontos de referência.

Na Medicina Humana, essa demarcação dos lobos já está descrita e é utilizada na rotina de exames, permitindo a localização das lesões e facilitando a comunicação entre ultra-sonografistas e cirurgiões por meio da padronização dos segmentos hepáticos. Os pontos de referência anatômica para essa segmentação são os ramos portais e veias hepáticas, além de estruturas como ligamentos, fissuras e a vesícula biliar (VALLE, ${ }^{8} 1993$; VEZOZZO et al., ${ }^{9} 1993$ ).

Segundo Lafortune et al. ${ }^{10}$ (1991), que descrevem a segmentação hepática ultra-sonográfica do fígado humano, o estudo dos segmentos hepáticos e a identificação de seus lobos tornam as avaliações pré-cirúrgicas e o controle de lesões, únicas ou múltiplas, mais precisos.

A presente pesquisa ${ }^{11}$ teve como objetivo a identificação das veias hepáticas e vasos portais por meio da ultra-sonografia, avaliando-se a possibilidade de uma forma de determinação dos lobos hepáticos de cães com base naqueles vasos, de maneira semelhante à segmentação de fígados humanos proposta por Lafortune et al. ${ }^{10}$ (1991) e também descrita por Valle ${ }^{8}$ (1993) e Vezozzo et al. ${ }^{9}$ (1993).

\section{Material e Método}

No presente estudo foram utilizados 16 cães adultos de raças variadas, sendo 15 vivos e 1 cadáver, todos provenientes do Hospital Veterinário da Faculdade de Medicina Veterinária e Zootecnia da Universidade de São Paulo (HOVET - FMVZ/USP), São Paulo, SP. Os 15 vivos foram selecionados da rotina do Setor de Radiologia da FMVZ/USP, 10 ( 5 machos e 5 fêmeas, com peso entre 8 e $45 \mathrm{~kg}$ ) porque não apresentavam sinais clínicos de enfermidades que pudessem comprometer o fígado de alguma maneira e com fígado sem alterações ultrasonográficas (ecogenicidade normal, ecotextura homogênea, sem a presença de nódulos, massas ou cistos no parênquima, margem regular e tamanho normal); e os outros 5 ( 3 machos e 2 fềmeas, pesando entre 8 e $14 \mathrm{~kg}$ ) porque apresentavam congestão venosa direita, sendo esta e suas conseqüências como hepatomegalia generalizada com aumento de calibre de vasos sanguíneos hepáticos - as únicas alterações presentes.

O cadáver foi utilizado para obtenção de molde vascular em vinil (Vinyl - Union Carbide Corporation Chemical and Plastic, New York, U.S.A.) que permitiu a observação da topografia dos vasos portais e das veias hepáticas do parênquima lobar. 
Este molde, por ser de material rígido, tem a vantagem de manter a forma tridimensional dos vasos dentro de cada lobo hepático, conservando suas relações. Para este propósito, foi utilizado o fígado de um cão da raça Husky Siberiano, do sexo masculino, sem idade conhecida, que veio a óbito sem apresentar sinais de doença hepática, sendo seu fígado injetado in loco (Figura 1).

Para os exames ultra-sonográficos, os cães foram preparados com jejum alimentar de 12 horas, tricotomia da região correspondente à cavidade abdominal e aplicação de gel específico (Multi Gel ${ }^{\circledR}$ - Indústria e Comércio de Produtos Gelatinosos Ltda.) sobre a pele. Durante os exames, foram posicionados em decúbito dorsal sobre calha de espuma, sendo rotacionados para a direita ou esquerda quando necessário. A contenção foi realizada sem auxílio de anestésicos (Figura 2).

O aparelho utilizado foi o TOKIMEC CS $3030^{\circledR}$ com transdutor convexo de 5,0 MHz e linear de 7,5 MHz.

Realizou-se o procedimento descrito por Probst e Kunzel ${ }^{12}$ (1993), Wu e Carlisle ${ }^{13}$ (1995) e Mwanza et al. ${ }^{14}$ (1996), identificando-se, inicialmente, as veias porta e cava caudal, junto ao hilo hepático, para que se pudesse seguir os vasos do sistema porta e as veias hepáticas, respectivamente. A seguir, foram realizados cortes ultra-sonográficos longitudinais e transversais, nas regiões subcostal e intercostal, conforme a necessidade de deslocamento ou rotação do transdutor para o acompanhamento do trajeto de cada estrutura vascular, visibilizada desde a região do hilo hepático até a periferia do órgão.

A diferenciação entre os vasos portais e as veias hepáticas foi realizada da maneira descrita por Nyland et al. ${ }^{2}$ (1995), Nyland e Park ${ }^{3}$ (1983), Nyland e Hager ${ }^{4}$ (1985), Wrigley ${ }^{5}$ (1985), Lamb $^{7}$ (1990 e 1991), Probst e Kunzel $^{12}$ (1993), e Partington e Biller ${ }^{1,15}$ (1995 e 1996), sendo considerados ramos portais os vasos com paredes ecogênicas e, veias hepáticas, vasos sem paredes ecogênicas.

Depois dos exames dos cães sem alteração hepática foram utilizados outros com congestão venosa direita, a fim de confirmar os vasos estudados, uma vez que, nesses processos, os segundos apresentaram-se mais visíveis. Seguiu-se o mesmo procedimento realizado anteriormente para a realização dos exames.

Os exames ultra-sonográficos foram registrados por meio de gravação em vídeo e fotos em filme fotográfico Ektascan $M^{\circledR} 20 \times 25 \mathrm{~cm}$. Os filmes facilitaram a identificação dos vasos por permitirem inúmeras reavaliações do exame de cada animal posteriormente.

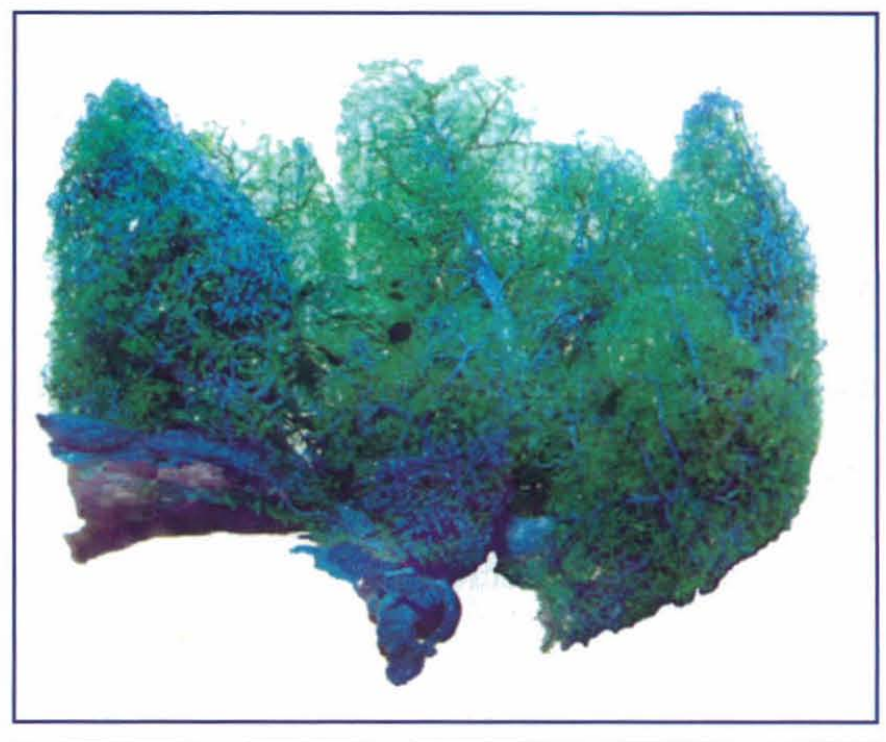

Figura 1 - Molde em vinil de veias hepáticas (azul) e sistema porta hepático (verde). (FMVZ/USP, 2002)

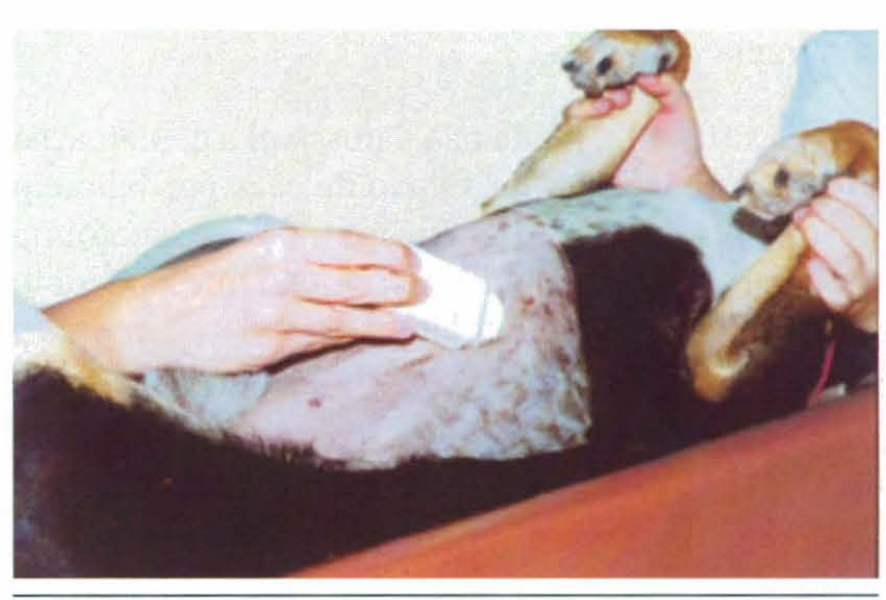

Figura 2 - Exame em decúbito dorsal, com o animal sobre calha de espuma e tricotomizado adequadamente para o procedimento. (FMVZ/USP, 2002) 


\section{Resultados}

\section{Identificação das Veias Hepáticas}

A veia cava caudal serviu como principal referência para localização das outras. Foi observada em corte longitudinal e em corte transversal, com o transdutor orientado, respectivamente, longitudinal e transversalmente em relação ao animal. Para a obtenção dessas imagens, tal dispositivo foi posicionado nos espaços intercostais direitos ou na região subcostal xifóide (Figura 3).

A veia hepática esquerda apresentou grande calibre, menor apenas que o da veia cava caudal, onde desemboca. Foi visibilizada próximo ao diafragma em corte longitudinal, posicionando-se o transdutor na região subcostal xifóide, orientado transversalmente em relação ao animal (Figura 4).

A veia hepática média que drena os lobos quadrado e medial direito, foi identificada drenando na veia hepática esquerda, bem próximo à veia cava caudal, com o transdutor na região subcostal xifóide, orientado transversalmente ou levemente oblíquo em relação ao animal (Figura 5).

Os vasos venosos dos lobos quadrado e medial direito foram observados ao lado da vesícula biliar, unindo-se dorsalmente e formando a veia hepática média. $\mathrm{O}$ transdutor foi posicionado na região subcostal xifóide, orientado transversalmente ou levemente oblíquo em relação ao animal.

As veias do lobo lateral direito e processo caudado do lobo caudado foram identificadas drenando na veia cava caudal. O transdutor posicionou-se nos espaços intercostais direitos, em região paravertebral, orientado longitudinalmente, com o animal rotacionado para a esquerda na calha de espuma. Para sua identificação, seguiu-se a veia cava caudal em direção caudal desde o diafragma. $\mathrm{O}$ primeiro tronco ou conjunto de veias a drenar na veia cava caudal corresponde aos vasos do lobo lateral direito e, o segundo aos do processo caudado do lobo caudado (Figura 6).

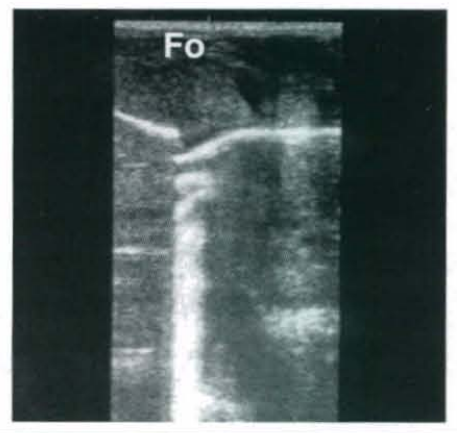

Figura 3 - Imagem ultra-sonográfica do forame da veia cava caudal no diafragma (Fo). (FMVZ/USP, 2002)

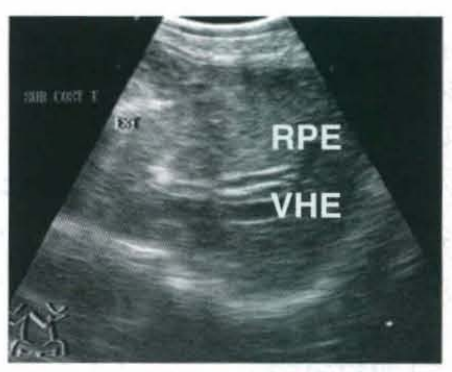

Figura 4 - Imagem ultra-sonográfica do ramo portal esquerdo (RPE) apresentando paredes ecogênicas, paralelo à veia hepática esquerda (VHE). (FMVZ/USP, 2002)

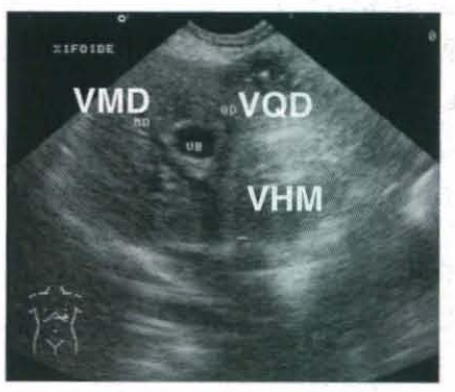

Figura 5 - Imagem ultra-sonográfica da veia hepática média (VHM) formada pela confluência da veia hepática do lobo medial direito (VMD) e da veia hepática do lobo quadrado (VQD). (FMVZ/USP, 2002)

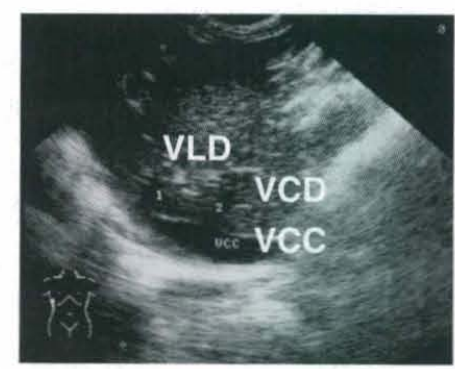

Figura 6 - Imagem ultra-sonográfica da veia cava caudal (VCC), veia hepática do lobo lateral direito (VLD) e veia hepática do processo caudado (VCD). (FMVZ/USP, 2002) 
Tanto nos animais normais como nos que apresentavam congestão venosa hepática não foi possível a identificação completa das veias de todos os lobos isoladamente. Por exemplo, embora visibilizadas, não foi possível diferenciar as veias que drenam o lobo lateral esquerdo das que drenam o lobo medial esquerdo, pela sobreposição das estruturas. A veia do processo papilar do lobo caudado não foi identificada devido à localização do estômago ventralmente a esse processo, interferindo na obtenção de sua imagem (Figura 7).

As veias hepáticas esquerdas, média e as dos lobos quadrado e medial direito apresentaram calibre bem aumentado nos animais com congestão venosa hepática em comparação aos cães sem alterações circulatórias, sendo facilmente identificadas nesses últimos. (Figura 7).

\section{Sistema Porta Hepático}

A veia porta foi observada em corte longitudinal com o transdutor orientado longitudinalmente em relação ao animal e em corte transversal com o transdutor orientado transversalmente em relação ao animal. Para a obtenção dessas imagens, o transdutor foi posicionado nos espaços intercostais direitos ou na região subcostal xifóide.

O ramo portal esquerdo foi identificado apresentando trajeto relativamente paralelo à veia hepática esquerda, ramificando-se da veia porta, próximo ao colo da vesícula biliar. Posicionou-se o transdutor na região subcostal xifóide, orientado transversalmente em relação ao animal (Figura 4).

O ramo portal direito foi identificado com o transdutor na região intercostal, paravertebral direita, rotacionando-se o animal para a esquerda sobre a calha. Esse vaso ramifica-se da veia porta, e pode ser visibilizado em corte transversal, orientando-se o transdutor longitudinalmente em relação ao animal, e em corte longitudinal orientando-se o transdutor transversalmente.

O ramo portal do lobo medial direito foi identificado à direita da vesícula biliar, com o transdutor posicionado na região subcostal xifóide e orientado transversalmente em relação ao animal.
Quanto aos lobos medial esquerdo e lateral esquerdo, alguns segmentos foram observados com o transdutor orientado transversalmente na região subcostal xifóide, porém, não foi possível seguir seu trajeto e nem identificá-los.

Não foram localizados os ramos portais dos lobos quadrado, lateral direito e processo caudado do lobo caudado. Muitos segmentos desses vasos portais puderam ser observados distribuídos pelo parênquima hepático, contudo, não foi possível acompanhar seus trajetos e, portanto, detectá-los.

O ramo portal do processo papilar do lobo caudado também não foi identificado devido à localização do estômago ventralmente a esse processo, interferindo na obtenção de sua imagem.

No que se refere aos vasos do sistema porta hepático, não foi notada diferença entre o calibre dos vasos portais dos cães que apresentaram congestão venosa direita em comparação com os normais, no entanto, o figado aumentado facilitou o exame (Figura 8).

O molde em vinil foi de grande utilidade para orientação da topografia dos vasos, esclarecendo dúvidas durante o exame.

\section{Discussão}

As veias cava caudal e porta foram localizadas com facilidade, assim como ramos maiores das veias hepáticas. Todavia, em relação às veias hepáticas menores e ao sistema porta, com exceção da veia porta esquerda, encontrou-se grande dificuldade de identificação. Também foi possível afirmar - por terem sido utilizados alguns animais que apresentavam congestão venosa direita-que as veias hepáticas dilatadas facilitam essa tarefa, o que não ocorre em relação aos vasos portais, que não apresentaram diferença significante nos resultados obtidos entre os grupos de animais com e sem congestão venosa hepática.

As veias cava caudal, porta hepática e a região do hilo hepático foram localizadas facilmente próximas ao colo da vesícula biliar, da mesma maneira observada por outros autores (PROBST, KUNZEL, ${ }^{12}$ 
SALGADO, A. B.; MARIANA, A. N. B. Identificação de veias e vasos portais hepáticos de cães por ultra-sonografia. Ultrasonographic identification of canine liver portal vessels and hepatic veins. Identificación de venas y vasos portales hepáticos de perros por ultrasonografia. Rev. Educ. Contin. CRMV-SP / Contin. Educ. J. CRMV-SP, São Paulo, v. 8, n. 1, p. 82-92, 2005.

\section{VEIA CAVA CAUDAL E VEIAS HEPÁTICAS}

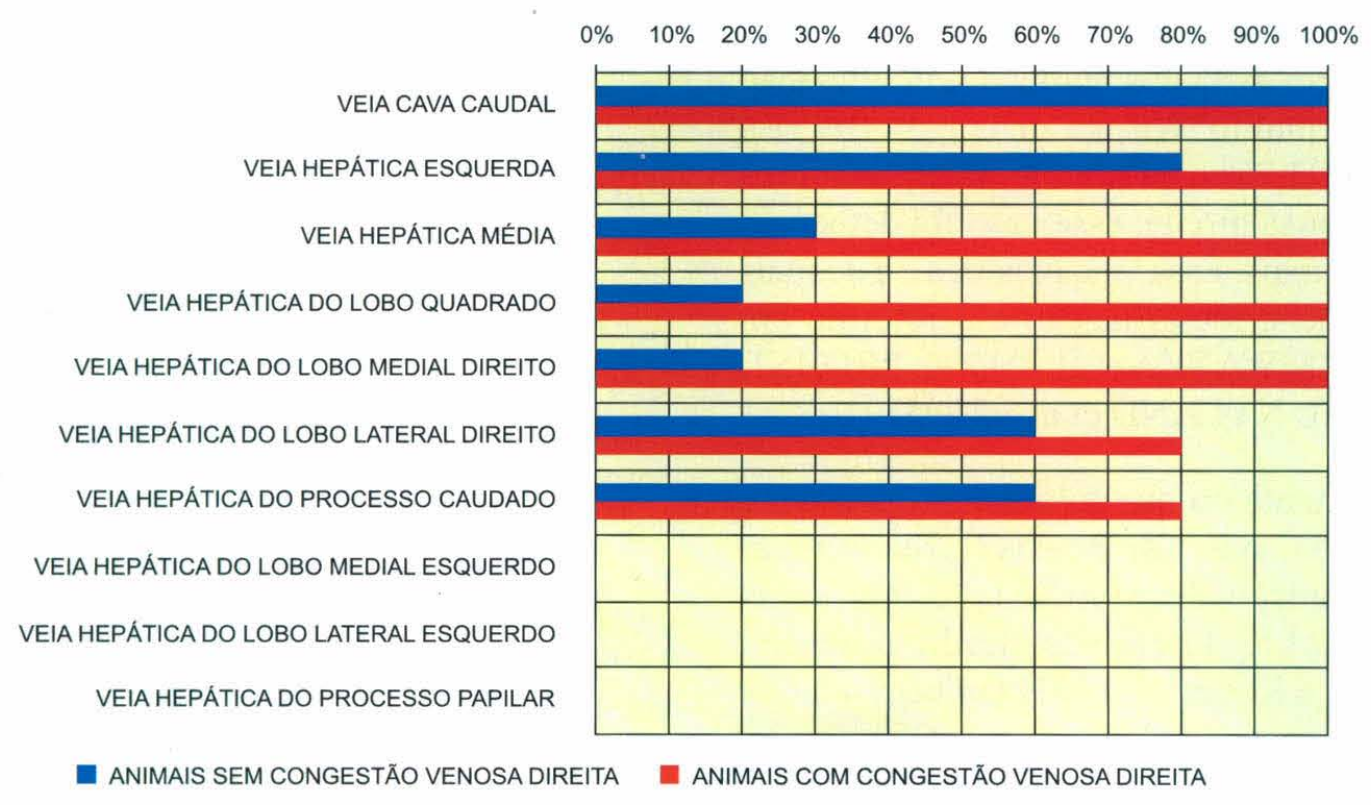

Figura 7 - Quadro comparativo da visualização, em porcentagem, entre a veia cava caudal e as veias hepáticas, nos grupos de animais com e sem alteração congestiva venosa direita (FMVZ/USP, 2002)

\section{SISTEMA PORTA HEPÁTICO}

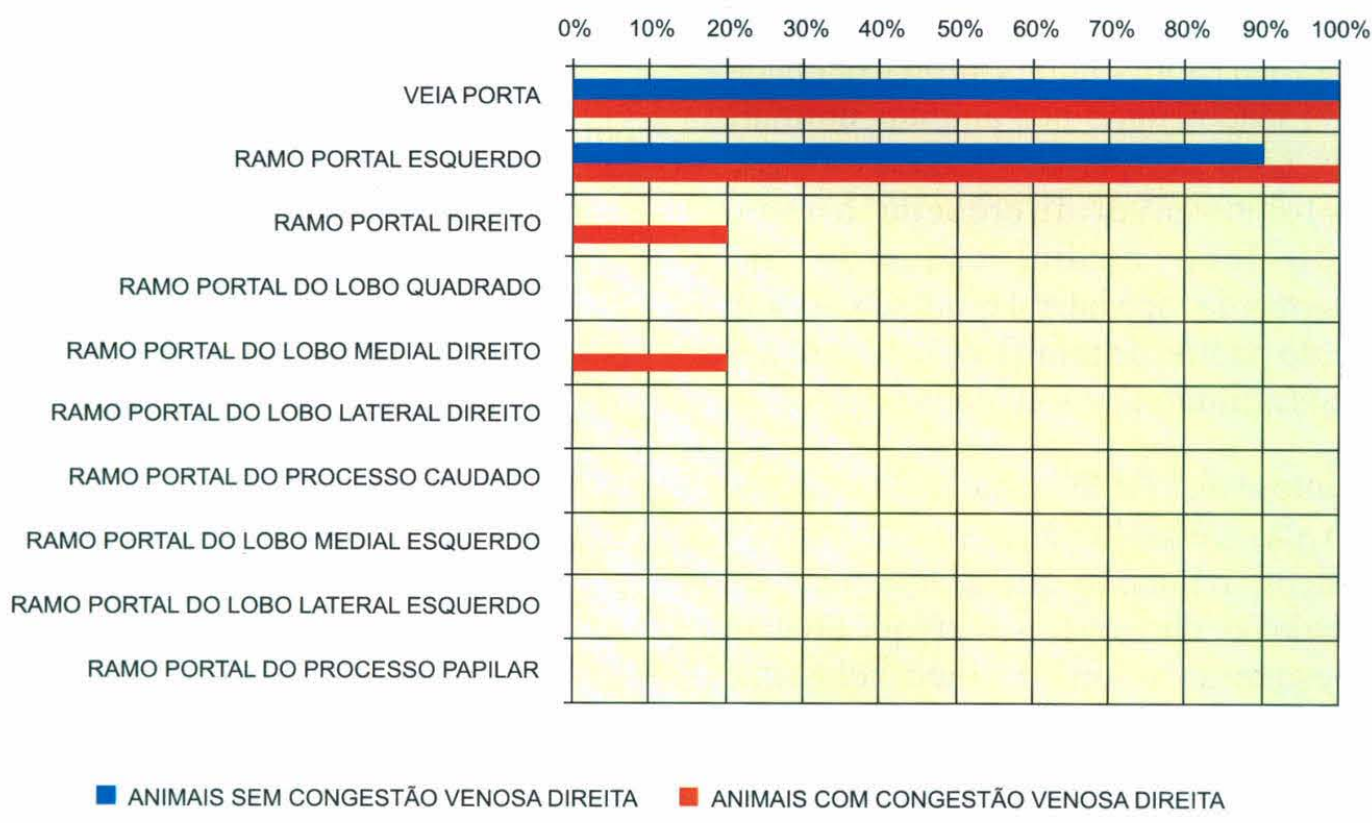

Figura 8 - Quadro comparativo da visualização, em porcentagem, da veia porta e seus ramos nos grupos de animais com e sem alteração congestiva venosa direita (FMVZ/USP, 2002) 
SALGADO, A. B.; MARIANA, A. N. B. Identificação de veias e vasos portais hepáticos de cães por ultra-sonografia. Ultrasonographic identification of canine liver portal vessels and hepatic veins. Identificación de venas y vasos portales hepáticos de perros por ultrasonografia. Rev. Educ. Contin. CRMV-SP/Contin. Educ. J. CRMV-SP, São Paulo, v. 8, n. 1, p. 82-92, 2005.

1993; NYLAND et al., ${ }^{2}$ 1995; WU, CARLISLE, ${ }^{13}$ 1995; MWANZA et al.. ${ }^{14}$ 1996).

Na presente pesquisa, a veia hepática esquerda foi identificada em $80 \%$ dos animais sem alteração circulatória, enquanto Wu e Carlisle ${ }^{13}$ (1995) a observaram em $93,75 \%$. Já em animais portadores de congestão venosa direita, esse vaso foi detectado em $100 \%$ dos animais. Essa veia hepática é a de mais fácil visibilização e identificação e é referida em outros trabalhos (MWANZA et al. ${ }^{14}$ 1996; PROBST, KUNZEL ${ }^{12}$ 1993; NYLAND et al., ${ }^{2}$ 1995).

Diferentemente do que foi relatado por Wu e Carlisle $^{13}$ (1995), não foi possível reconhecer facilmente o padrão de ramificação das veias hepáticas dos lobos lateral esquerdo e medial esquerdo. Probst e Kunzel ${ }^{12}$ (1993) também relatam terem identificado as veias desses lobos. Observaram dois grandes ramos, um mais cranial, que drena o lobo medial esquerdo e um mais caudal, que drena o lateral esquerdo, este, por sua vez, recebendo um ramo que vem de sua porção mais cranial e outro de sua porção mais caudal. Mwanza et al., ${ }^{14}$ (1996) referem ter observado as veias dos lobos médios e esquerdos unindo-se, formando a veia hepática esquerda. Entretanto, não descrevem precisamente a identificação das veias dos lobos medial e lateral esquerdo. Esses vasos apresentam calibre adequado para serem visibilizados tanto nos animais normais como naqueles com congestão venosa direita. Contudo, não foi possível diferenciar o ramo proveniente do lobo medial esquerdo das ramificações internas do lobo lateral esquerdo. Assim, a complexidade do padrão de ramificação foi um dos fatores que impossibilitaram a sua identificação.

Morais-Pinto et al. ${ }^{16}$ (1999) estudaram o padrão de ramificação das veias hepáticas por meio de moldes anatômicos, relatando que a veia hepática esquerda é formada por duas ou três veias que drenam o lobo lateral esquerdo e uma a cinco veias que drenam o medial esquerdo. Dessa maneira, associando-se à complexidade das ramificações e à variação do padrão de ramificação apresentada de animal para animal, obtêm-se os fatores que impedem a identificação ultra-sonográfica de tais vasos, pelo menos, pelas técnicas até hoje conhecidas.
A veia do lobo quadrado foi identificada à esquerda da vesícula biliar; e a do lobo medial direito à direita daquela, como referido por Wu e Carlisle ${ }^{13}$ (1995). Esses vasos foram observados, na presente pesquisa, em $20 \%$ dos animais normais e em $100 \%$ dos que apresentavam congestão venosa direita. Portanto, concluiu-se que, para que esses vasos possam ser identificados e seja possível seguir seu trajeto - observando sua confluência na veia hepática média até a veia hepática esquerda -, é necessário que seu calibre esteja aumentado. $O$ pequeno calibre desses vasos nos animais normais foi o fator que impossibilitou sua identificação.

Quanto às veias do lobo lateral direito e processo caudado do lobo caudado, Wu e Carlisle ${ }^{13}$ (1995) as detectaram em $100 \%$ de seus animais; no presente estudo foram observadas em $60 \%$ dos animais normais e em $80 \%$ dos animais que apresentavam congestão venosa direita. Probst e Kunzel $^{12}$ (1993) relatam que esses vasos são difíceis de serem visibilizados, não sendo possível seguir seu trajeto. Os resultados obtidos permitem discordar desses autores, pois aqueles vasos foram seguidos, na presente pesquisa, em $60 \%$ dos animais normais. Por outro lado, não se conseguiu o resultado de $100 \%$ como Wu e Carlisle ${ }^{13}$ (1995), nem ao examinar os animais com congestão venosa direita. Ressalte-se que o padrão de ramificação desses vasos não é complexo como o dos lobos esquerdos do fígado, e sua identificação é possível toda vez que puderem ser seguidos.

A veia porta esquerda foi visibilizada com facilidade da maneira referida por Probst e Kunzel ${ }^{12}$ (1993), Nyland et al. ${ }^{2}$ (1995) e Wu e Carlisle ${ }^{13}$ (1995), o que não ocorreu em relação aos outros vasos do sistema porta, que mostraram grande dificuldade para serem identificados.

Mwanza et al..$^{14}$ (1996) e Wu e Carlisle ${ }^{13}$ (1995) descrevem o reconhecimento do ramo portal direito sem citar em quantos animais ele foi observado. Nyland et al. ${ }^{2}$ (1995) e Mwanza et al..$^{14}$ (1996), ao referirem-se ao ramo portal direito, descrevem que este emite um ramo para a direita, irrigando os lobos lateral direito e medial direito. No entanto, segundo Wu e Carlisle ${ }^{13}$ (1995) e a Nomenclatura Anatômica Veterinária Ilustrada ${ }^{17}$ (1999), 
o ramo portal direito irriga o lobo lateral direito e o processo caudado do lobo caudado; e o ramo portal esquerdo, as regiões restantes, inclusive o lobo medial direito. Devido às divergências na descrição anatômica desse vaso, seguiu-se, na presente pesquisa, a descrição da Nomenclatura Anatômica Veterinária Ilustrada ${ }^{17}$ (1999), identificando-se, dessa maneira, o ramo portal direito em apenas um animal.

Não foram vistos os ramos portais do lobo lateral direito e processo caudado do lobo caudado. Wu e Carlisle ${ }^{13}$ (1995) mencionam sua visibilização seguindo a veia porta direita sem descrever o número de observações; também não dão referências mais detalhadas sobre o ramo portal do lobo medial direito, o qual citam que foi identificado em apenas um animal.

Os ramos portais dos lobos quadrado, medial esquerdo e lateral esquerdo não foram visibilizados com facilidade. Alguns animais até apresentaram parte dessa ramificação visível, porém, difícil de ser acompanhada por extensão suficiente a permitir seu reconhecimento. Logo, não se identificaram esses vasos em nenhum animal por dois motivos: a complexidade de seu padrão de ramificação, que impede a diferenciação entre um ramo interno do lobo lateral esquerdo de um do lobo medial esquerdo ou quadrado, e a impossibilidade de seguir seu trajeto, para certificação de que alguma ramificação importante não estaria sendo perdida. Devido a esses fatores, os resultados obtidos foram bastante diferentes dos de Wu e Carlisle ${ }^{13}$ (1995) com relação à percepção do ramo portal do lobo lateral esquerdo, por eles identificado em $93,75 \%$ dos animais e, o do medial esquerdo, $75 \%$ dos animais. Quanto ao ramo portal do lobo quadrado, não fazem referência ao número de observações, citando que o decúbito dorsal é o melhor para sua visibilização.

Concorda-se com Probst e Kunzel ${ }^{12}$ (1993) quando afirmam que os ramos portais podem ser observados ao exame sonográfico sendo, porém, sua identificação bem mais difícil que das veias hepáticas, talvez até impossível.

Mwanza et al. ${ }^{14}$ (1996) comparam a angiografia à ultra-sonografia, concluindo que o padrão de distribuição das veias hepáticas e do sistema porta pode ser visibilizado por ambos os métodos, dentre os quais a ultra-sonografia permite uma avaliação dinâmica e não-invasiva, sendo as técnicas complementares entre si. Citam que a principal desvantagem da ultra-sonografia está na impossibilidade de acompanhar o trajeto dos vasos sem reposicionar o transdutor, e que a presença de gás intestinal, ascite e a conformação corporal podem levar à perda de alguns dados. Esses e outros fatores como o tipo e a freqüência dos transdutores devem ser considerados ao realizar-se um exame para identificação de vasos hepáticos.

Com relação à freqüência dos transdutores, Wu e Carlisle ${ }^{13}$ (1995) relatam que devem ser escolhidos de acordo com o tamanho do animal, utilizando equipamentos de 7,5 MHz, de $5 \mathrm{MHz}$ e de 3,5 MHz. No presente trabalho, assim como no de Probst e Kunzel ${ }^{12}$ (1993), foram utilizados transdutores de 5 e 7,5 $\mathrm{MHz}$ de freqüência. Mwanza et al. ${ }^{14}$ (1996) utilizaram as três freqüências, e os animais pesavam entre $10 \mathrm{e} 14 \mathrm{~kg}$. Como no presente estudo não foi utilizado aquele de 3,5 MHz, e Probst e Kunzel ${ }^{12}$ (1993) e Wu e Carlisle ${ }^{13}$ (1995) não mencionaram o peso dos animais, não se tem base para comparação do quanto a freqüência dos transdutores pode ter influenciado nas diferenças entre os resultados obtidos.

Outro dado importante é o tipo de transdutor utilizado. De acordo com Nyland e $\mathrm{Hager}^{4}$ (1985) e Partington e Biller ${ }^{1,15}$ (1995 e 1996), os transdutores que mais se adaptam ao exame intercostal são os setoriais e os convexos. Concorda-se com esses autores, porque esses dispositivos, principalmente os setoriais, seguidos dos microconvexos, têm menor superfície de contato com a pele do animal quando comparados aos lineares, o que possibilita seu posicionamento no espaço intercostal sem sobreposição às costelas, evitando a sua sombra acústica. Probst e Kunzel ${ }^{12}$ (1993) e Mwanza et al. ${ }^{14}$ (1996) relatam ter utilizado transdutores setoriais, Wu e Carlisle ${ }^{13}$ (1995) não mencionaram nada a esse respeito. Para a realização da presente pesquisa, os transdutores utilizados foram um linear de 7,5 MHz, julgado inadequado ao exame intercostal e um 
convexo de $5 \mathrm{MHz}$, que permitiu a obtenção de imagens consideradas adequadas. Com o convexo, a sombra das costelas interferiu somente no exame de animais taquipnéicos, quando o fígado move-se muito rapidamente sob o gradil costal acompanhando os movimentos respiratórios.

Inicialmente, concordando com outros autores ${ }^{1-}$ 4,15,18-20 que relatam ser a sedação geralmente desnecessária, optou-se por não sedar ou anestesiar os animais, tendo sido realizado o exame ultrasonográfico com o paciente contido gentilmente. Porém, durante esta pesquisa, notou-se que a anestesia do animal poderia ser de grande valia, já que permite controlar os movimentos respiratórios. A taquipnéia dificulta a visibilização hepática, uma vez que, nessa condição, o fígado move-se rapidamente sob o gradil e a sombra das costelas impede o acompanhamento dos vasos para sua identificação, especialmente os de menor calibre. Desse modo, concordou-se posteriormente com Probst e Kunzel ${ }^{12}$ (1993) ao relatarem que a anestesia facilita o exame. Mwanza et al..$^{14}$ (1996) utilizaram a sedação quando julgaram necessária, todavia não comentam quantos animais foram submetidos a esse procedimento e se houve diferença entre o resultado dos dois grupos.

Observou-se ainda que a interferência dos movimentos respiratórios é menor em animais com congestão venosa direita devido à hepatomegalia e ao maior calibre das veias. O fígado aumentado permite que apenas poucas imagens tenham necessidade de serem realizadas em região intercostal. Isso explica o maior número de observações dos vasos portais em animais com congestão hepática direita, quando comparados aos normais, apesar de não ter sido notada diferença de calibre desses vasos entre os dois grupos estudados.

\section{Conclusões}

Baseado no modelo humano, não foi possível identificar os lobos hepáticos de cães, por meio da ultrasonografia, devido à dificuldade no reconhecimeto de muitas veias e vasos portais dos lobos hepáticos desses animais. Entretanto, os vasos devem sempre ser pesquisados, pois, quando passíveis de identificação, mostram-se importantes estruturas de referência.

\section{Abstract}

Objective: To identify the canine hepatic veins and portal hepatic vessels by ultrasonography, evaluating the possibility of hepatic lobe determination based on these vessels in dogs, similar to human liver segmentation. Ultrasonography is a good method for evaluation of the liver, giving information on size, shape and parenchymal changes. Although the visualization and differentiation between hepatic veins and portal vessels is possible in dogs, their anatomical identification is difficult and the literature does not describe any methods for hepatic lobe determination. Material and Method: Ultrasound scans were done in 15 live animals - 10 healthy dogs and 5 dogs presenting with right venous congestion. A cadaver was employed for obtainance of a vinyl vascular cast. Results and Conclusions: Our results were documented by means of videos and photographs and showed that many of the hepatic veins and vessels of the portohepatic system are difficult to identify, leading to the conclusion that the human model of segmentation is not adequate for use in dogs. Still, whenever identified these vessels can serve as an aid in lobe determination, once they are important reference anatomical structures.

Keywords: Anatomy. Liver. Hepatic veins. Ultrasonography. Dogs. 
SALGADO, A. B.; MARIANA, A. N. B. Identificação de veias e vasos portais hepáticos de cães por ultra-sonografia. Ultrasonographic identification of canine liver portal vessels and hepatic veins. Identificación de venas y vasos portales hepáticos de perros por ultrasonografia. Rev. Educ. Contin. CRMV-SP/Contin. Educ. J. CRMV-SP, São Paulo, v. 8, n. 1, p. 82-92, 2005.

\section{Resumen}

Objetivo: Identificar las venas y vasos portales hepáticos de perros a través de ultrasonografia, evaluándose la posibilidad de determinar los lóbulos hepáticos de perros con base en esos vasos de manera semejante a la segmentación de hígados humanos. El examen ultrasonográfico es un buen método para la evaluación del hígado proveyendo datos sobre el tamaño, forma y alteraciones del parénquima. En perros, aunque la visualización y diferenciación entre las venas hepáticas y vasos portales sea posible, son de dificil identificación anatómica y no hay descripción en la literatura de ningún método que permita determinar los lóbulos hepáticos. Material y Método: Se utilizaron 15 perros vivos para la realización de exámenes ultrasonográficos, 10 sanos y 5 con congestión venosa derecha. Se utilizó un cadáver para la obtención del molde vascular en vinilo. Resultados y Conclusiones: Los resultados se registraron en vídeos y fotos y mostraron que muchas de las venas hepáticas y vasos del sistema porta hepático, son de dificil identificación, lo que permite concluir que el modelo humano de segmentación es inadecuado para la utilización en perros. A pesar de ello, siempre que los vasos sean identificados pueden ser utilizados como auxilio para determinación de los lóbulos, pues representan importantes estructuras anatómicas de referencia.

Palabras-clave: Anatomía. Hígado. Venas hepáticas. Ultrasonografía. Perros.

\section{Referências}

1. PARTINGTON, B. P; BILleR, D. S. Hepatic imaging with radiology and ultrasound. Veterinary Clinics of North America: Small Animal Practice, v. 25, p. 305-335, 1995.

2. NYLAND, T. G.; MATTOON, J. S.; WISNER, E. R. Ultrasonography of the liver. In: NYLAND, T. G.; MATTOON, J. S. Veterinary diagnostic ultrasound. Philadelphia: Saunders, 1995. p. 52-73.

3. NYLAND, T. G.; PARK, R. D. Hepatic ultrasonography in the dog. Veterinary Radiology, v. 24, p. $74-84,1983$.

4. NYLAND, T. G.; HAGER, D.A. Sonography of the liver, gallbladder, and spleen. Veterinary Clinics of North America: Small Animal Practice, v. 15, p. 1123-1148, 1985.

5. WRIGLEY, R. H. Radiographic and ultrasonographic diagnosis of liver diseases in dogs and cats. Veterinary Clinics of North America: Small Animal Practice, v. 15, p. 21-38, 1985.

6. LAMB, C. R. Abdominal ultrasonography in small animals: examination of the liver, spleen and pancreas. Journal of Small Animal Practice, v. 31 , p. $6-15,1990$.
7. LAMB, C. R. Ultrasonography of the liver and biliary tract. Problems in Veterinary Medicine, v. 3, p. $555-573,1991$.

8. VALLE, P. V. Anatomia In: CERRI, G. G.; ROCHA, D. C. Ultra-sonografia abdominal. São Paulo: Sarvier, 1993. p. $31-90$

9. VEZOZZO,D.C.P.; ROCHA,D.C.; CERRIG.G. Fígado. In: CERRI, G. G.; ROCHA, D. C. Ultra-sonografia abdominal. São Paulo: Sarvier, 1993. p. 61-90

10. LAFORTUNE, M. et al. Segmental anatomy of the liver: a sonographic approach to the Couinaud nomenclature. Radiology, v. 181, p. 443-448, 1991.

11. SALGADO, A. B.; MARIANA, A. N. B. Identificação das regiões correspondentes aos lobos hepáticos de cães por meio da ultrasonografia. 2002. $83 \mathrm{f}$. Dissertação (Mestrado em Anatomia dos Animais Domésticos)- Faculdade de Medicina Veterinária e Zootecnia da Universidade de São Paulo, São Paulo, 2002.

12. PROBST, A.; KUNZEL, W. Anatomische Grundlagen zur sonographischen Differenzierung der Leberlappen des Hundes. Wierner. Tierarztliche Monatschrift, v. 80 , p. $200-2007,1993$. 
13. WU, J.; CARLISLE C. H. Ultrasonographic examination of the canine liver based on recognition of hepatic and portal veins. Veterinary Radiology and Ultrasound, v. 36, p. 234-239, 1995.

14. MWANZA, T. et al. Ultrasonography and angiographic examination of normal canine liver vessels. Japanese Journal of Veterinary Research, v. 44, p. 179-188, 1996.

15. PARTINGTON, B. P.; BILlER, D. S. Liver. In: GREEN, R. W. Small animal ultrasound. Philadelphia: Lippincot Williams and Wilkins, 1996. p. 105-130.

16. MORAIS-PINTO, L. Estudo anatômico das veias hepáticas em cães sem raça definida (Cannis familiaris - Linnaeus, 1758). 1999. 66 f. Dissertação (Mestrado em Anatomia dos Animais Domésticos)-
Faculdade de Medicina Veterinária e Zootecnia, Universidade de São Paulo, São Paulo, 1999.

17. SCHALLER, O. (Ed.) Nomenclatura anatômica veterinária ilustrada. São Paulo: Manole, 1999.

18. BUNCH, S. E. Hepatobiliary and exocrine pancreatic disorders. In: NELSON, R. W.; COUTO, C. G. Essential of small animal internal medicine. Saint Louis: Mosby Year Book, 1992. p. 367-445.

19. SELCER, B. A. The liver and gallbladder. In: CARTEE, R. E. et al. Practical veterinary ultrasound. Philadelphia: Williams and Wilkins, 1995. p. 88-106.

20. BILLER, D. S.; BLACKWELDER, T. Hepatic ultrasonography: a valuable tool in small animals. Veterinary Medicine, v. 39, p. 646-653, 1998. 